\title{
Inhalt des 214. Bandes.
}

\author{
Erstes Heft (10. Oktober).
}

Seite

II. Yokoyama, Yugo, Uber die Wirkung des Thymus im Organismus. (Aus dem Pathologischen Institut des Auguste-Viktoria-Krankenhauses



III. Adler, Leo. Thymus und Adrenalsystem. (Aus dem Pathologischen Institut des Auguste-Viktoria-Krankenhauses zu Berlin-Schöneberg.)

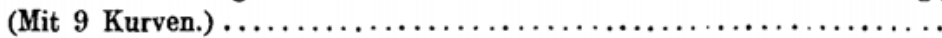

IV. Ceelen, W., Ober Karnifikation in tuberkulösen Lungen. (Aus dem Pathologischen Institut der Universität Berlin.) (Hierzu Tafel I und II.)

V. Sehrt, Ernst, Uber das Vorkommen einer doppeltlichtbrechenden Substanz als normaler Bestandteil der Prostataepithelzelle des Menschen

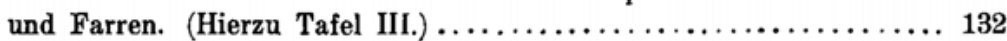

VI. Anitschkow, N., Zur Kenntnis der malignen Neuroblastome des N. sympathicus. (Aus dem Pathologischen Institut in Straßburg i. E.) (Hierzu 7 Textfiguren.).............................. 137

VII. $\mathrm{O} \mathrm{k}$ a, Dr., Zur Histologie der Vinylaminnephritis. (Aus dem Pathologischen Institut der Universität Freiburg i. Br.) (Hierzu Tafel IV.).......... 149

\section{Zweites Heft (12. November).}

VIII. Wengraf, Fritz, Zur Kenntnis des sogenannten embryonalen Adenosarkom der Niere. (Aus dem Pathologischen Institut der Landeskrankenanstalt in Brünn.) (Hierzu 8 Mikrophotogramme.) ............. 161

IX. K o ch, Karl, Beiträge zur Pathologie der Bauchspeicheldrüse. (Aus dem Pathologischen Institut der Universität Berlin) .............. 180

X. Hada, Benzo, Uber die Gehirnkomplikationen des Keuchhustens mit besonderer Berücksichtigung der "Pachymeningitis productiva interna“. (Aus dem Pathologisch-Anatomischen Institut der Universtät Berlin.) 206

XI. Scagliosi, G., Beitrag zur Ätiologie des Duodenalgeschwüres (akzessorisches Nebenpankreas, Duodenaldrüsenadenom und -adenokarzinom). (Aus dem Pathologischen Institut des Hauptkrankenhauses St. Johann und der Stadt Turin.) (Hierzu 7 Textfiguren.) ................. 220

XII. Schepelmann, Emil, Experimente zur plastischen Aszitesradinage, zugleich ein Beitrag zur Histologie implantierter FormolgefäBe. ( $\Lambda$ us 
der chirurgischen und orthopädischen Privatklinik von Dr. Voeckler und Dr. Schepelmann in Halle a. S.) (Hierzu 6 Textfiguren und Taf. V.) 279

XIII. Merkel, Hermann, Zur Kenntnis der Aneurysmen im Bereich der Arteria hepatica (dissezierendes Aneurysma mit Ausheilung durch totale Thrombose mit sekundärer Verkalkung und Verknöcherung des Thrombus). (Aus dem Pathologischen Institut der Universität Erlangen.) (Hierzu 5 Textfiguren.).............................. 289

XIV. Heigel, A., Ober eine besondere Form von Entwicklungsstörung der Trikuspidalklappe. (Aus dem Pathologisch-anatomischen Institut der deutschen Universität Prag.) (Hierzu 1 Textfigur.) ................ 301

XV. Unn a, P. G., Die Herkunft der Plasmazellen .................. 320

Drittes Heft (17. Dezember).

XV. Unn a, P. G., Die Herkunft der Plasmazellen. (Hierzu Tafel VI u. VII.)

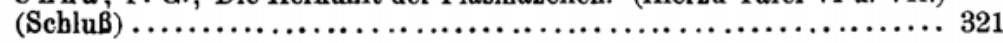

XVI. Wätjen, J., Uber das Vorkommen von Plasmazellen und ihre Bedeutung bei Pneumonien des Kindesalters nach akuten Infektionskrankheiten. (Aus dem Pathologischen Institut der Universität Freiburg i. Br.). (Hierzu 1 Textfigur.) ............................... 340

XVII. Stilling, H., und Beitzke, H., Uber Uterostumoren bei Kaninchen. (Aus dem Pathologischen Institut in Lausanne.) (Hierzu 6 Textfiguren.) 358

XVIII. Boeckmann, Ein Beitrag zur Ätiologie der Pachymeningitis interna haemorrhagica. (Aus dem Pathologischen Institut des AugustaHospitals zu Berlin.) ................................. 380

XIX. Wohlwill, Fr., Uber Pachyminingitis haemorrhagica interna. Aus dem Pathologischen Institut des Allgemeinen Krankenhauses Hamburg-

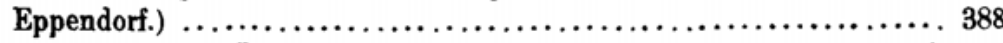

XX. A bramow, S., Ober die Veränderungen der Hypophyse bei der experimentellen Diphtherie. (Aus dem Pathologisch-anatomischen Institut der Universität Moskau und dem Institut des Dr. Ph. M. Blumenthal.) (Hierzu 2 Textfiguren.) ............................... 408

XXI. Mühlmann, M., Das Nervenpigment beim Papagei. (Hierzu 1 Textfigur 412

XXII. Finzi, Otello, Über Veränderungen der Magenschleimhaut bei Tieren nach Nebennierenexstirpation und über experimentell erzeugte Magengeschwüre. (Aus der Chirurgischen Klinik der Universität Pisa.) (Hierzu



XXIII. Ljubimowa, W. J., Ein Fall von Ulcus ventriculi verursacht durch Schimmelpilze. (Aus dem Prosektorium des Marienhospitals zu St. Peters-



XXIV. Meulengracht, E., Uber die Gastritis polyposa. (Hierzu 5 Textfiguren.) 438

XXV. Wetzel, Erwin, Beitrag zur Lehre von der Malakoplakie der Harnblase. (Aus dem Pathologischen Institut in Straßburg i. E.)....... 450

XXVI. Kalbermatten, J. de, Beobachtungen über Glykogen in der glatten Muskulatur. (Aus dem Pathologischen Institut der Universität Bern.) 455

XXVII. Joest, E, und Emshoff, E., Nachtrag zu unserer Arbeit ${ }_{n}$ Studien über die Histogenese der Lymphdrüsentuberkulose und die Frühstadien des Lymphdrüsentuberkels“. 